\title{
LOS HUMEDALES ANTE EL CAMBIO CLIMÁTICO*
}

\author{
Bárbaro V. Moya \\ Centro Meteorológico Provincial. CITMA Matanzas \\ Ana E. Hernández \\ Centro de Información y Gestión Tecnológica. CITMA. Matanzas \\ Héctor Elizalde Borrell \\ Centro Meteorológico Provincial. CITMA Matanzas
}

\section{RESUMEN}

Las emisiones antropogénicas y naturales a la atmósfera, al aumento de concentración de gases de efecto invernadero, el calentamiento global, el cambio climático y el cambio global son hoy situaciones muy preocupantes para la comunidad científica internacional.

Las vulnerabilidades, los impactos y las medidas de adaptación a escenarios futuros del clima, son una prioridad para la que ya vienen trabajando científicos y especialistas.

Los humedales, ecosistemas con vulnerabilidad natural a los embates del clima, según el tercer informe del IPCC, han sido poco estudiados en su relación con el cambio del clima. Recientemente Ramsar ha solicitado al IPCC el desarrollo de un informe técnico sobre la relación de los humedales y el cambio climático, sin embargo, existe hoy una gama de conocimientos, que a pesar de las incertidumbres, permite tener un acercamiento a la acción del cambio climático sobre los humedales.

En el presente estudio se muestra un análisis preliminar sobre vulnerabilidades, impactos y medidas de adaptación de los humedales al cambio climático.

Palabras clave: Cambio climático, calentamiento global, mitigación, vulnerabilidad, impactos, adaptación.

\section{ABSTRACT}

Natural and antropogenic emissions to the atmosphere, the growth of the green house gases concentration, global warming, climate and global change are today, one of the most important situations to be worried about by the international scientist community.

The vulnerability, impacts and adaptation measures to future climate scenarios, are the priority goal for scientist and specialist working on it.

The wetlands, ecosystems with natural vulnerability to the impact of climate, according to third Report of IPCC, have been low level studied in its interaction with the cli-

\footnotetext{
* El presente trabajo, forma parte de una conferencia impartida en el Simposio Internacional Humedales 2003. Ciénaga de Zapata. Matanzas. Cuba.
} 
mate change. Recently, Ramsar asked to IPCC the develop of technical report about the relationship existing between wetlands and climate change, however, there is a stock of knowledge, although uncertainty, give the chance to know about the climate change impacts to the wetlands.

The current study shows a preliminary analyse about vulnerability, impacts and adaptation of the wetlands to the climate change. tation.

Key words: Climate change, global warming, mitigation, vulnerability, impacts, adap-

\section{Introducción}

La emisión de gases a la atmósfera producto de la actividad del hombre ha provocado afectaciones sensibles al medio atmosférico. Para la mayor parte de la comunidad científica internacional la más importante de estas afectaciones se relaciona con el aumento de gases de efecto invernadero y un aumento del potencial de retención del calor en la atmósfera. Esto ha estado ocurriendo paralelamente a un aumento de la temperatura media global en las últimas decenas, que si bien en el decenio 1981-90 resultaba alarmante, entre 1991 y 2000 alcanzó valores aún más preocupantes, ocurriendo en la misma 8 de los 10 años más calientes de los últimos 140 años para aquel momento, entre ellos el 1998, que ha resultado el año con mayor valor de temperatura media global, desde que se tienen registros de medición o monitoreo confiables. El presente siglo XXI, nos ha aportado ya el 2002 como segundo año más caliente y el 2003 que según la Organización Meteorológica Mundial es el tercer año caliente en la serie citada anteriormente.

Este calentamiento global se asocia a una serie de anomalías en el comportamiento del clima en casi todo el mundo. El aumento de la temperatura, el crecimiento del nivel del mar, la mayor ocurrencia de sequías e inundaciones, son algunos de los fenómenos que más han llamado la atención en los últimos años.

Esta situación coloca a la comunidad científica internacional ante la interrogante de si el clima está cambiando. Respuestas a favor y en contra se suceden, aunque ya existe consenso que el planeta enfrentará escenarios climáticos diferentes de los actuales en el futuro. Para ello el mundo trabaja ya, principalmente bajo la tutoría del Panel Intergubernamental de Cambio Climático (IPCC, siglas en inglés) y otras instituciones internacionales en estrategias de adaptación a estos escenarios futuros.

Los impactos de este cambio en el clima van a afectar sensiblemente la sociedad, la economía y el medioambiente. No obstante existe una gran incertidumbre en qué realmente ocurrirá, fundamentalmente en la respuesta que tendrá la atmósfera ante este proceso de calentamiento y qué impactos provocará este sobrecalentamiento.

La humanidad trabaja hoy en la adaptación a estos escenarios por venir, y actualmente una de las más recomendadas estrategias de adaptación consiste en determinar diferentes escenarios de trabajo, analizar y evaluar las vulnerabilidades, determinar los impactos y a partir de esta información practicar medidas concretas de adaptación.

Los humedales por sus características y peculiaridades suelen ser ecosistemas sensibles a ser afectados por las variaciones del clima y los fenómenos extremos del tiempo. Grandes pérdidas, a veces irreversibles, suelen ocurrir en estos por la acción del clima y los fenómenos meteorológicos extremos. Cambios en la temperatura, en los patrones de precipitación, aumento del nivel del mar, son entre otras variaciones del clima que pueden producir sensibles impactos en los humedales. 
El presente trabajo desarrolla un análisis de la relación entre los humedales y el cambio climático.

\section{Desarrollo}

Algunos humedales, muestran una elevada vulnerabilidad al cambio climático, debido entre otras características a la fragilidad de sus ecosistemas, fragilidad que se ve estimulada tanto por acciones naturales como antropogénicas. No obstante esta vulnerabilidad, la relación entre los humedales y el cambio climático debe verse en dos aristas principales:

1. El humedal y su potencial para la mitigación del cambio climático y la acción de eventos meteorológicos extremos.

2. El humedal y su vulnerabilidad ante el cambio climático. Los impactos y medidas de adaptación al cambio climático.

\subsection{Mitigación del cambio climático}

En el primer caso los humedales, según el concepto promulgado en Ramsar (2000), sirven de sumidero al 40\% (Ramsar, 2000) del carbono que se genera en el planeta. La destrucción de estos evitaría la eliminación de importantes cantidades de gases de efecto invernadero y por ende aumentaría el potencial de retención del calor en la atmósfera, con implicación en el calentamiento global y el crecimiento de las temperaturas con todo su efecto sobre el sistema climático en primera instancia y sobre la vida en una óptica más amplia.

Dentro de la propiedad de mitigación del cambio climático, en este caso a los impactos que produce este cambio, los humedales tienen como funciones la estabilización de costas, así como constituyen una primera línea de defensa ante la acción de fenómenos severos del tiempo, permitiendo el desgaste de estos y la disminución de la intensidad de estos cuando actúan sobre otras áreas.

Entre los mecanismos de mitigación a los impactos del cambio climático, que tienen los humedales podemos citar:

- Estabilización de costas.

- Regulan la cantidad y calidad del agua (Colchones hidrológicos).

- Constituyen una primera defensa a la acción de huracanes y tormentas severas.

- Disminuyen el impacto por fuertes vientos.

- Fuente de recursos para consumo directo.

\subsection{Vulnerabilidad, impactos y adaptación al cambio climático}

\subsubsection{Vulnerabilidad}

En el segundo caso los humedales constituyen ecosistemas frágiles, con especies con endemismo local y otras peculiaridades naturales y antropogénicas que lo hacen muy vulnerables al cambio climático. Entre estas vulnerabilidades cabe citar:

- Poca altura sobre el nivel del mar o exposición a inundaciones o invasiones de agua.

- Exposición a los eventos de sequías.

- Asentamientos humanos en zonas bajas o expuestas a invasiones de agua. 
- Exposición de las fuentes de abastos de agua potable a la inclusión salina o al desbalance hídrico.

- La exposición, endemismo local y fragilidad de la flora y fauna.

- Mal manejo del humedal y fragmentación de ecosistemas

- Fragilidad de los ecosistemas.

- Exposición a tormentas y fenómenos severos del tiempo.

- Incertidumbres en las respuestas de la atmósfera al aumento de las concentraciones de gases de efecto invernadero y sobre los impactos por el cambio climático.

- Escaso desarrollo de estrategias de adaptación y mitigación de impactos por cambio y variabilidad del clima en los humedales.

Como muestra de la elevada vulnerabilidad de estos ecosistemas al cambio climático, debe mencionarse lo recogido en el tercer informe de evaluación el Grupo Intergubernamental de Expertos sobre el Cambio Climático (IPCC) que concluyó que algunos humedales incluidos los arrecifes, atolones y manglares, y los de praderas, así como los ecosistemas de bosques tropicales y boreales, y los ecosistemas árticos y alpinos, con inclusión del permafrost, figuran entre los sistemas naturales especialmente vulnerables al cambio climático debido a su escasa capacidad de adaptación, y que pueden sufrir daños apreciables e irreversibles.(IPCC, 2001)

\subsubsection{Impactos}

Una serie de impactos potenciales pudieran ocurrir en los humedales por el cambio climático. El derretimiento de los permafrost en los Alpes por ejemplo, puede provocar afectaciones a la biodiversidad que se desarrolla bajo estos humedales, que pudiera llegar hasta la pérdida de especies por la imposibilidad de la emigración, dado la topografía de la región. Los cambios en el régimen de temperaturas, radiación, viento e hidrología en los ecosistemas acuáticos pueden afectar directamente la disponibilidad de nutrientes en el ecosistema, la subsistencia, crecimiento y reproducción de los organismos, y la producción del ecosistema.

Algunos impactos potenciales esperados son:

- Afectación en las funciones ecológicas, al provocar cambios en la hidrología y otras condiciones físicas y químicas que favorecen la convivencia entre los componentes bióticos y abióticos del ecosistema.

- Pérdidas de hábitat y otras afectaciones a la flora y la fauna.

- Afectaciones por sequías y eventos de extremas precipitaciones (pérdidas sociales, económicas y medioambientales).

- Afectaciones a la fuente de abasto de agua por inclusión salina o desbalance hídrico.

- Aumento de la erosión costera.

- Afectaciones a ecosistemas bajo permafrosts.

- Afectaciones al transporte de sedimentos y nutrientes.

- Variaciones en los patrones epidemiológicos y de las epifititas fundamentalmente por cambios en las condiciones medioambientales donde se desarrollan los patógenos.

\subsubsection{Adaptación}


Teniendo en cuenta lo visto en las vulnerabilidades y los impactos resalta la necesidad de tomar medidas de adaptación al cambio climático en los humedales, como vía para impedir su desaparición o mitigar los impactos que sobre estos pueden ocurrir asociados al cambio climático. Entre estas medidas de adaptación destacamos:

- Desarrollo de estrategias conjuntas entre los investigadores, especialistas y tomadores de decisión, para la adaptación y mitigación de los impactos por CC.

- Restaurar y rehabilitar las zonas de humedales, eliminando el stress presente en los humedales y disminuyendo su vulnerabilidad.

- Evitar nuevas presiones que reduzcan la capacidad de los humedales a responder al cambio climático.

- Prevenir la fragmentación de los humedales.

- Protección y disminución de población en los lugares más bajos o vulnerables.

- Desarrollo de estudios de evaluaciones de vulnerabilidad, impactos y medidas adaptación, cuyos resultados sirvan de base para el trabajo de los administradores de humedales y para el manejo de estos.

- Monitoreo ecológico y climático.

\section{Conclusiones}

- El clima ha estado variando en los últimos años y la tendencia en su comportamiento actual es a seguir variando. Los modelos sobre comportamiento futuro del clima, indican que el clima cambiará. Esto llevará a importantes anomalías y cambios en el comportamiento del mismo, con una serie de impactos sobre el medio, por los que es importante prepararse para los futuros escenarios climáticos y desde ahora preparar medidas de adaptación.

- Los humedales son especialmente vulnerables al cambio climático, por lo que requieren una atención especial y el desarrollo de una estrategia de adaptación. Esta vulnerabilidad es aún mayor si se tiene en cuenta las peculiaridades, características físico-geográficas e importancia ecológica de gran parte de los humedales.

- Los impactos por cambio climático, provocarán sensibles afectaciones a los humedales, en muchos casos irreversibles.

- Una buena estrategia de adaptación para los humedales resulta la determinación y evaluación de sus vulnerabilidades, la determinación de los impactos potenciales y una vez desarrollado el análisis relacional vulnerabilidad - impactos la toma de medidas de adaptación.

- La adaptación de los humedales al cambio climático es una necesidad que deben resolver de conjunto la comunidad científica y los administradores y tomadores de decisión de estos ecosistemas.

\section{Bibliografía}

ALFONSO, A. et al (2002): Informe final del Proyecto Territorial Desarrollo de un sistema de Manejo del Humedal Ciénaga de Zapata. Proyecto Territorial. CITMA. Matanzas. p. 167. IPCC (2001): Climate Change 2001. Impacts, adaptation and vulnerability. IPCC, WMO, UNEP. Cambridge University Press. U.K p. 1009.

MOYA, B. (2002): «Climate Change in Varadero one of the most important touristic places in Caribbean sea», en IHDP. Workshop 2002 Bonn. Alemania pp. 3-7. 
RAMSAR (1999): Los humedales y el cambio climático. Examen de la colaboración entre la Convención sobre los Humedales (RAMSAR, Irán 1971) y la Convención Marco de las Naciones Unidas sobre el Cambio Climático.www.ramsar.org/key_unfccc_bkgd.htm.

RAMSAR (2000): Notas informativas sobre los valores y las funciones de los humedales. Mitigación del cambio climático. Hoja informativa N5 2000 www.ramsar.org/values_climate_s.htm.

RAMSAR (2002): $8^{a}$ Reunión de la Conferencia de las Partes Contratantes en la Convención sobre los Humedales (Ramsar, Irán,1971), Valencia, España, 18 al 26 de Noviembre de 2002, p. 4. 